\title{
74. MARCHESINIA MACKAII (HOOK.) S. GRAY Y NOWELLIA CURVIFOLIA (DICKS.) MITT. (HEPATICAE) EN EL NOROESTE PENINSULAR
}

\author{
Juan REINOSO FRANCO y $\mathrm{M}^{\mathrm{a}}$ del Carmen VIERA BENITEZ
}

Marchesinia mackaii (Hook.)S.Gray and Nowellia curvifolia (Dicks.)Mitt. in the North-West Peninsular

Palabras clave. Briófitos, Corología, Galicia, España.

Key words. Bryophytes, Chorology, Galicia, Spain.

Con esta nota damos noticia de recolecciones efectuadas en la provincia de $\mathrm{A}$ Coruña de dos hepáticas, de distribución rara en la Península Ibérica, y que suponen una ampliación de su areal conocido para Galicia (fig. 1). Nuestras citas representan las localidades peninsulares más occidentales y se emplazan en el horizonte eucolino, del piso colino del subsector Galaico-Asturiano septentrional, sector Galaico-Asturiano de la provincia Cántabro-Atlántica, Región Eurosiberiana, según la síntesis corológica de Rivas Martínez (1987).

\section{Marchesinia mackaii (Hook.) S. Gray.}

Hs, A CORUÑA: Cedeira, Vixía Herbeira, 29TNJ84, sobre sustrato serpentinícola en claros de brezal de Ulici gallii-Ericetum mackaianae ericetosum vagantis, en acantilados sobre el Oceáno Atlántico, 400 m, 18-5-1995, J. Reinoso \& M. C. Viera, SANTbryo.

Marchesinia mackaii es una interesante hepática de distribución atlántico subtropical, que penetra en el dominio mediterráneo occidental gracias a topoclimas principalmente caracterizados por una humedad atmosférica constante. Si se exceptúa la especie tipo, $M$. mackaii, la distribución del género está limitada a las regiones tropicales de América Latina y Africa (Geissler, 1990). Conocida en el norte de España en Asturias, Cantabria, País Vasco y Navarra (Casas et al. 1989). En el Mediterráneo se encuentra en las estaciones disyuntas de
Algeciras y Tarragona. Crece en la Europa atlántica, desde Escocia hasta Portugal y Macaronesia, y es frecuente en la parte occidental de las islas británicas, Francia y fachada tirrénica de Italia (Salanon et al. 1990). La primera cita de M. mackaii en Galicia se debe a Buch (1937) en la localidad pontevedresa de El Grove, cerca de la isla de La Toja. Cincuenta años después añadimos un nuevo registro en Galicia (Reinoso, 1985), al que hay que agregar la nueva estación coruñesa ahora señalada, en donde $M$. mackaii presenta un comportamiento ecológico diferente al observado por nosotros hasta el momento, poblaciones casi monoespecíficas sobre cortezas de Laurus nobilis. En la localidad de

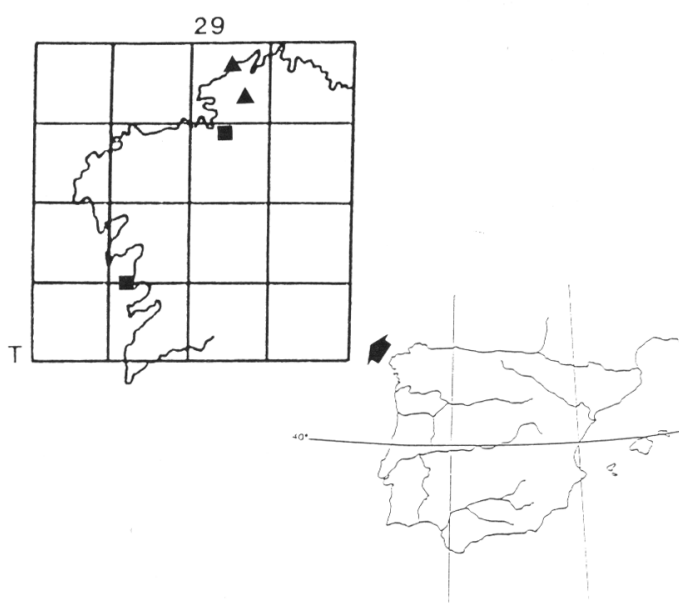

Fig. 1.- Distribución de Nowellia curvifolia y Marchesinia mackaii en Galicia: conocidas. $\Delta$ Nuevas localidades. 
Cedeira, el taxon denunciado se desarrolla como epilítico aerohigrófilo en uno de los dos núcleos serpentínicos de Galicia, asociado a Frullania fragilifolia y Plagiochila exigua, sobre sustrato ultrabásico deficitario en sílice de tipo piroxenitas, dunitas y peridotitas serpentinizadas con baja relación $\mathrm{Ca} / \mathrm{Mg}$.

Nowellia curvifolia (Dicks.) Mitt.

Hs, A CORUÑA: Ortigueira, Serra da Capelada, cuenca del río Seixo 29TNJ83, sobre madera de castaño en descomposición en restos de Blechno-Quercetum roboris pulmonarietosum longifoliae, $80 \mathrm{~m}, 4-3-1989$, J. Reinoso, SANT-bryo.

Nowellia curvifolia es un taxon suboceánico-montano, considerado muy raro y conocido en escasos enclaves del País Vasco, Navarra, Huesca y Lleida y ausente del resto de la Península Ibérica (Casas et al. 1985). Su distribución en Europa es muy amplia, extendiéndose hasta Azores y Madeira; igualmente conocida en Asia y America (Schuster, 1974). La única referencia de esta especie en Galicia fué dada a conocer por nosotros (Reinoso, 1985), recolectada en bosque atlántico, donde existe una rica flora mesófila e hidrófila de Querco-Fagetea, con diferentes aportes hídricos que contribuyen a la persistencia de una humedad atmosférica muy elevada. Según nuestras observaciones $N$. curvifolia es una especie de comportamiento meso-esciófilo, que vive siempre sobre madera muy húmeda y en estado final de descomposición, al abrigo de estaciones boscosas con gran humedad, en la proximidad de arroyos. Siempre formando céspedes bien desarrollados y dando lugar a una comunidad muy característica formada por diferentes hepáticas como Bazzania tricrenata, Odontoschisma denudatum, Tritomaria exsectiformis y Lepidozia reptans, entre otras. La abundancia con que se encuentra en esta otra localidad de Ortigueira, mucho mayor que en la primera, hace disminuir notablemente el peligro de desaparición de esta interesante hepática epixílica acidófila.

AGRADECIMIENTOS. Agradecemos a la Dra. Carlota García Paz (Departamento de Edafología y Química Agrícola, Universidad de Santiago) la ayuda prestada en los datos geológicos.

\section{BIBLIOGRAFÍA}

BUCH, H. - 1934/1937- Muscinées récoltées dans le Nord-Ouest de la Péninsule Ibérique. Rev. Bryol. Lichénol. 7:238-248; 9:47-54. Paris.

CASAS, C., T. BRUGES, R.M. CROS y C. SERGIO -1985- Cartografía de Briófitos. Fasc. I: 1-50. Institut d'Estudis Catalans. Barcelona.

CASAS, C., T. BRUGES, R.M. CROS y C. SERGIO -1989- Cartografía de Briófitos. Fasc. II: 51100. Institut d'Estudies Catalans. Barcelona.

GEISSLER, P. -1990- Brioecología y briogeoghrafía. Enfoque y aplicación. Anales Jard. Bot. Madrid 46 (2): 353-358.

REINOSO, J. - 1985- Contribución al conocimiento de la flora briológica de Galicia. Briófitos de la fraga de Caaveiro, II. Hepáticas. Acta Bot. Malacitana 10:17-26.

RIVAS-MARTÍNEZ, S. -1987- Memoria del mapa de las series de vegetación de España. 11-52. ICONA.

SALANON, R., J. GANDIOLI, P. GEISSLER. 1990-Marchesinia mackaii (Hook.) S.Gray dans les Alpes-Maritimes: étude floristique et écologique de sa station; posibilités de sauvegarde?. Cryptogamie, Bryol. Lichénol. 1990, $11(3): 283-298$.

SCHUSTER, R. -1974- The Hepaticae and Anthocerotae of North America. Vol. 3. Columbia University Press. New York.

Aceptado para su publicación en Mayo de 1998

Dirección de los autores. Departamento de Biología Vegetal (Botánica), Facultad de Biología, Universidad de Santiago de Compostela, 15706 Santiago de Compostela. España. 